SECTION 26. Radio-technique. Electronics. Telecommunications.

Gennadii Gennadyevich Miroshnikov

Candidate of Technical Science, Associate Professor

Astrakhan State University

ggmiroshnikov@gmail.com

\title{
AN EXPERIMENTAL APPROACH TO THE USE OF PROXY SERVERS IN TIME- SHIFTED TV
}

Abstract: The Internet Protocol Television services (IPTV) highly popular nowadays are based on IP protocol which supports broadcast and unicast services such as the Broadcasting of TV channels, Video On Demand and Time-Shifted Television. Distribution of individual television streams to viewers imply demands of considerable bandwidth and this could be challenging for network operators and telecommunication companies. The use of caching proxy servers could be a solution to this. This paper illustrates an experimental approach which showed high efficiency in proxy servers providing Time-shifted television allowing customers to watch content regardless of the time of broadcast.

Key words: Internet Protocol Television, caching, distributed servers, multicast, unicast, streaming IP-video, Time-Shifted TV, data rate, interactive television.

Time-shifted television, one of the most promising services within IPTV, is becoming increasingly popular mostly because it provides with a powerful and highly valued tool: customization [1-3].

The most attractive feature of a Time-Shifted TV architecture is that it adds a dedicated server and a data centre to complement an existing scheme of IPTV. The basic principle of its organization is that a subscriber has access a server which stores recorded content whilst are still being broadcast or already ended. Storing a large archive of television programmes over long periods necessarily means a significant increase of server capacity and a subsequent higher cost of implementation. On the contrary, Time-Shifted TV services can be provided on the same existing network infrastructure which is in use for broadcasting over IP using a multicast channels architecture [4-5]. A typical model of Time shifted TV added architecture is described on the Figure 1.

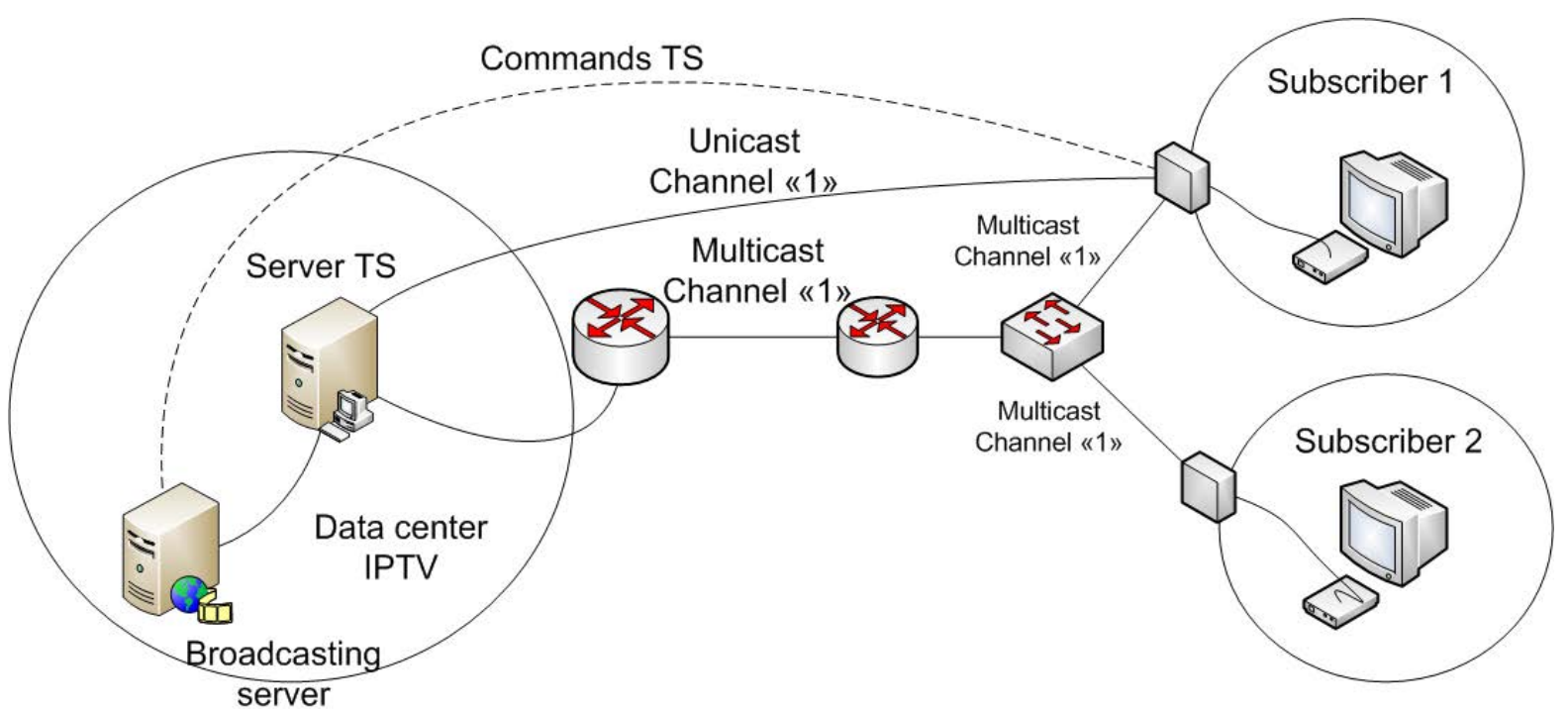

Figure 1 - An architectural model of Time Shifted TV added to an existing Multicast infrastructure. 
As seen above, Subscriber 1 could any time whitdraw content directly from the Time shifted TV dedicated server utilising a Unicast stream channel "1" even long after the broadcast of stream channel "1" has ended in Multicast mode [6]. This is made possible because content was copied simultaneously to a storage device directly available to set-top boxes so they could access the "shifted" version at a later stage.

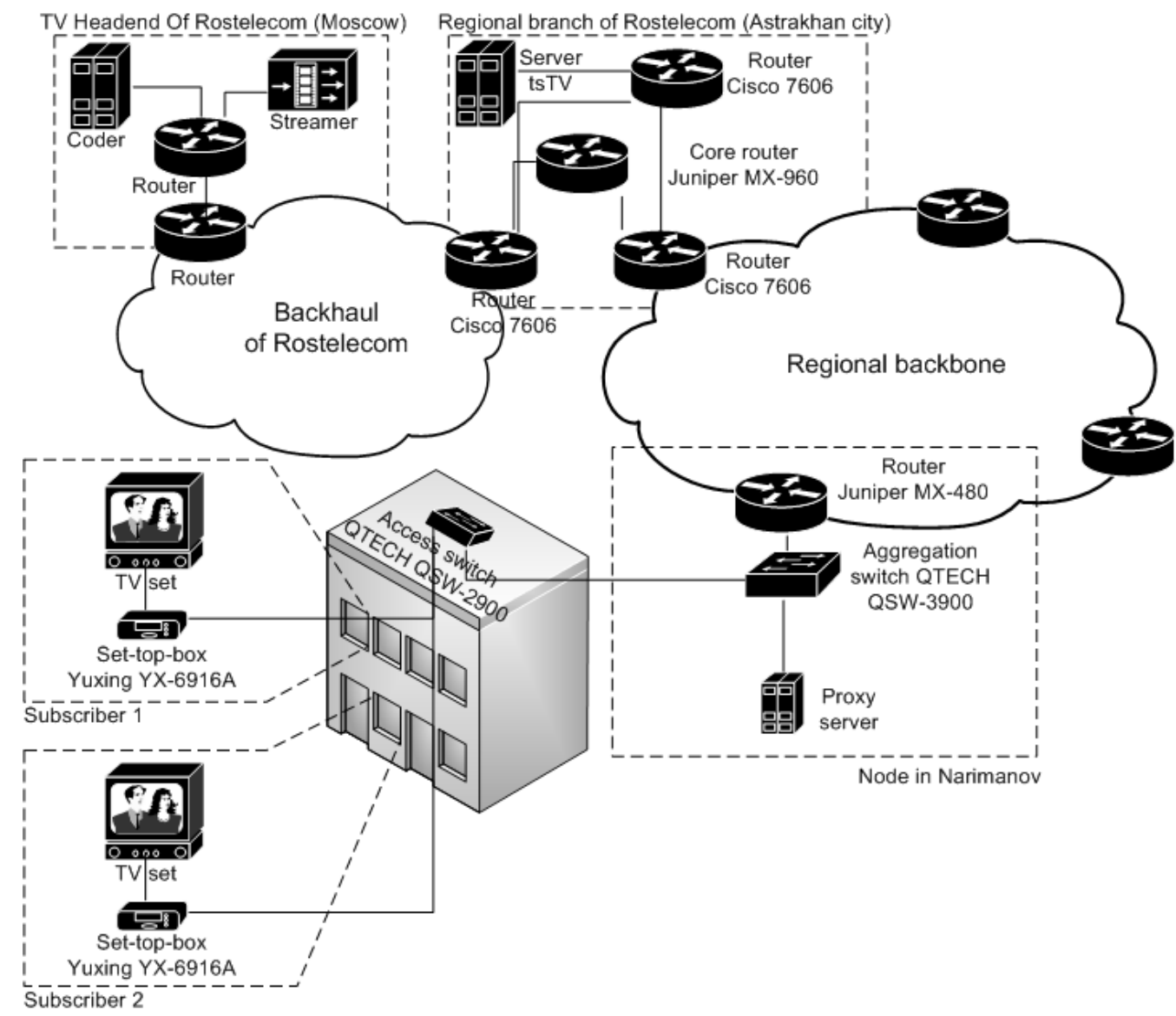

Figure 2 - Topology of a segment on the testing network used in Narimanov.

Under these conditions, Subscriber 2 can only access the content of channel "1" in real time since there is no direct connection to the Time-Shifted TV server.

Storing stream segments on a dedicated server also allows end-users additional functionalities such as pause, rewind and fast forward content. It is important to note, however, that a Time-shifted TV server may not need be a single physical part of hardware. It could be composed, in some configurations, by a streaming server IP directly fed data from the central IPTV or be distributed across multiple other servers. To illustrate the latter, a series of simulations were made at the town of Narimanov on a fragment of the network that belongs to OJSC "Rostelecom" in the Astrakhan region of the Russian Federation. This fragment included a Metro Ethernet access network [7-9] covering 79 homes. It's topology is shown in the Figure 2.

During the simulation the load on the data link between the aggregation node in Narimanov and the central communication node in Astrakhan city (where the time-shifted TV server is located) was measured. These measurements were made using the utility Iperf, and only traffic of the time-shifted TV services was measured [10]. Measurements were carried out in three stages. Firstly, without the use of proxy servers, later using a single proxy server and finally using two proxy servers in a co-operative caching mode. 


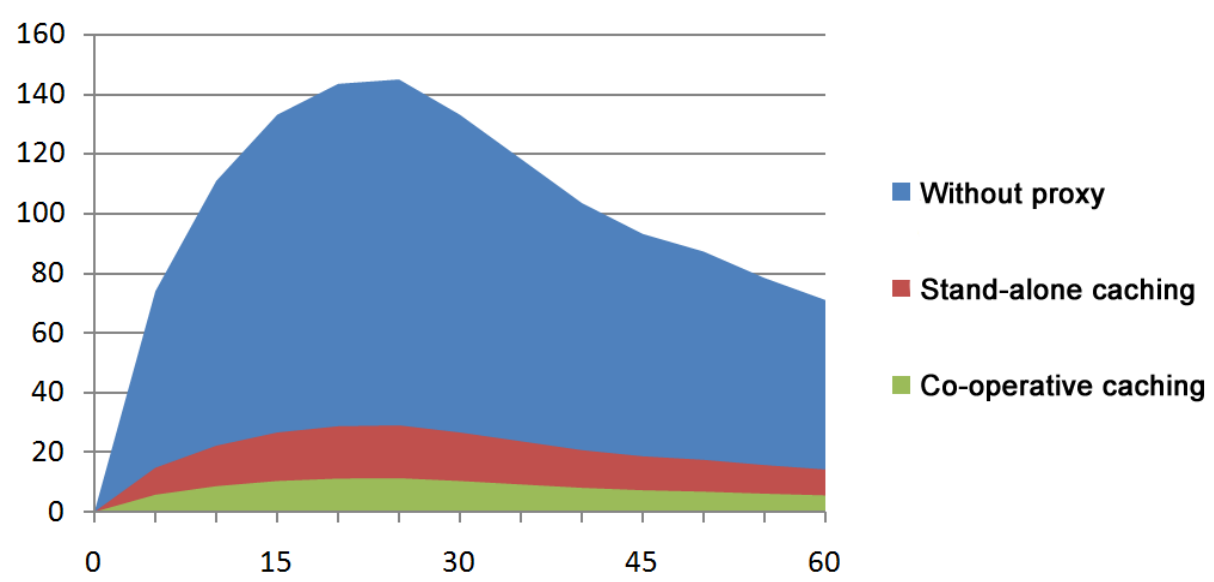

Figure 3 - Measurements of the loading link (in Mbps) between the aggregation node and the central node taken during the Narimanov's testing under three conditions.

These tests have shown that the use of proxy servers for caching the individual segments of video service in Time-shifted TV allowed it to reduce the load of the central server services and the data transmission channel between the access network and the backbone network provider. These measurements indicated also that co-operating proxy servers could offload the backbone of a data network between the aggregation node and the node of the installation in a central server Time-shifted TV more than five times in average.

\section{References:}

1. Bester J., Guna J., Kos A., Volk M. Quality-assured provisioning of IPTV services within the NGN environment / Bester J., Guna J., Kos A., Volk M. // IEEE Communications Magazine. - 2008. - no. 5. - p. 18-23. ISSN 0163-6804

2. Gunn H.J. The Basics of IPTV / Gunn H.J. - New York: IEC. 2007. - 189p. ISBN 9781931695589

3. Held G. Understanding IPTV / Held G. - New York: Auerbach Publications. 2006. 208p. ISBN 978-0849374159

4. Hens F.J. Triple Play: Building the converged network for IP, VoIP and IPTV / Hens F.J. - New York: Wiley-Interscience. 2008. - 416p. ISBN 978-0470753675

5. Han S., Lisle S., Nehib G. IPTV transport architecture alternatives and economic considerations / Han S., Lisle S., Nehib G. // IEEE Communications Magazine. - 2008. no. 2. - p. 70-77. ISSN 0163-6804

6. Kozierok C. The TCP/IP Guide: A Comprehensive, Illustrated Internet Protocols Reference / Kozierok C. - New York: No Starch Press. 2005. - 1616p. ISBN 9781593270476

7. Joseph V. Deploying Next Generation Multicast-enabled Applications: Label Switched Multicast for MPLS VPNs, VPLS, and Wholesale Ethernet / Joseph V. - New York: Morgan Kaufmann. 2011. - 560p. ISBN 978-0123849236

8. Hanrahan H. Network Convergence: Services, Applications, Transport, and Operations Support / Hanrahan H. - New York: Wiley. 2007. - 464p. ISBN 978-0470024416

9. Jain S., Hayward M., Kumar S. Broadband Infrastructure: The Ultimate Guide to Building and Delivering OSS/BSS from Businessedge Solutions / Jain S., Hayward M., Kumar S. - New York: Springer. 2003. - 304p. ISBN 978-1402073786

10. Hjelm J. Why IPTV: Interactivity, Technologies, Services (Telecoms Explained) / Hjelm J. - New York: Wiley-IEEE Press. 2008. - 370p. ISBN 978-0470998052

УДК 53.06 